\title{
Monitoreo de especies forestales nativas altamente presionadas en la cuenca del río Salaquí, municipio de Riosucio, Chocó, como estrategia para reducir su agotamiento progresivo
}

\author{
Monitoring of native forest species in highly pressured Salaquí River \\ basin Chocó Riosucio as a strategy to reduce its progressive depletion
}

\section{Robinson Stewart Mosquera M.*}

\section{Resumen}

Los bosques naturales tropicales del Darién chocoano, han sido objeto de explotación y sometidos a una fuerte presión antrópica, debido al aumento progresivo de las actividades productivas en la zona, lo que ha afectado fuertemente la estabilidad ecosistémica y por ende la distribución, abundancia y diversidad de algunas especies arbóreas que revisten de tanta importancia que son necesarias para mantener el equilibrio en una masa forestal determinada; por tal razón es indispensable la realización de estudios encaminados a la protección y seguimiento de especies forestales en condiciones de vulnerabilidad y amenaza, para lo cual el Instituto de Investigaciones Ambientales del Pacifico (IIAP) se ha propuesto brindar criterios específicos para el manejo sostenible, la protección y recuperación de estos bosques a través de la realización de estudios que contribuyan a cuantificar el estado ambiental del bosque y el grado de amenaza al que están expuestas las especies forestales allí presentes. Con base en lo anterior se desarrolló una investigación que estableció 6 parcelas temporales de muestreo de $50 \mathrm{~m} \times 50 \mathrm{~m}=2500 \mathrm{~m}^{2}(0.25 \mathrm{ha})$, muestreando los árboles de las especies seleccionadas con un diámetro a la altura del pecho $(D A P) \geq 10 \mathrm{~cm}$, distribuidas en 4 consejos comunitarios locales de la cuenca del río Salaquí, en el municipio de Riosucio. Se encontraron 372 especies, correspondientes a 7 familias y 13 géneros, con distribución variable entre parcelas; los resultados obtenidos presentan una serie de medidas de conservación inmediatas a tener en cuenta para garantizar la perpetuación y preservación de los bosques en mención.

Palabras clave: Darién, Especies amenazadas, Estabilidad ecosistémica, Manejo sostenible, Riosucio.

\begin{abstract}
Tropical natural forests Chocó Darién, have been exploited and subjected to strong human pressure, due to the progressive increase of productive activities in the area, which has strongly affected ecosystem stability and hence the distribution, abundance and diversity of some tree species that are of so much importance that are needed to maintain balance in a forest particular, for this reason it is essential to carry out studies aimed at the protection and monitoring offorest species in conditions of vulnerability and threat, for which environmental Research Institute of the Pacific (IIAP) has been proposed to provide specific criteria for sustainable management, protection and recovery of these forests through studies that help to quantify the environmental status of the forest and the degree of threat to which they are exposed forest species present. Based on
\end{abstract}

* Ingeniero Agroforestal, Contratista Instituto de Investigaciones Ambientales del Pacífico (IIAP).

e-mail: robinsonmosquera@gmail.com

Recibido: 23 de marzo de 2012

Aceptado: 25 de mayo de 2012 
the above research was undertaken, which established six temporary sampling plots of $50 \mathrm{~m} \times 50 \mathrm{~m}=2,500 \mathrm{~m}^{2}$ equivalent to 0.25 ha , sampling trees of selected species with a diameter at breast height $(D B H) \geq 10 \mathrm{~cm}$; in 4 local community boards river Basin Salaquí in Riosucio. 372 species were found, corresponding to 7 families and 13 genera, with variable between plots and the results obtained have a number of immediate conservation measures to be considered to ensure the perpetuation and preservation of forests in question.

Keywords: Darién, Ecosystem stability, Endangered species, Riosucio, Sustainable management.

\section{Introducción}

En el departamento del Chocó en las últimas décadas han aumentado los diferentes métodos de aprovechamiento de las especies maderables, lo cual contribuye a la destrucción de los bosques, situación que da como resultado la pérdida de la diversidad y la degradación de los ecosistemas. Por estas condiciones se pueden observar grandes volúmenes de madera que se están movilizando actualmente en todas las regiones del departamento y en especial en la zona del Darién Chocoano. Esto produce la necesidad de buscar alternativas para el control de la explotación de las especies forestales que se encuentran en peligro de agotamiento y asegurar así que los volúmenes comercializados correspondan a las especies autorizadas por la autoridad ambiental competente (CODECHOCO). De acuerdo con lo manifestado por investigadores como Gentry (1989) y García et al. (2004) se registra en el departamento del Chocó la presencia de gran número de especies forestales con diferentes grados de amenaza como es el caso del abarco (Cariniana pyriformis), chanul (Humiriastrum procerum), jigua negro (Ocotea cernea), guayaquil (Centrolobium paraence), caoba (Swietenia macrophylla) entre otras, lo que obliga al conocimiento preciso de esa realidad para buscar alternativas que promuevan la protección de estas especies. El Instituto de Investigaciones Ambientales del Pacífico (IIAP) y la Corporación Autónoma Regional para el Desarrollo Sostenible del Chocó (CODECHOCO), bajo los preceptos de un convenio interadministrativo, para adelantar con recursos del Fondo de Compensación Ambiental, el proyecto «Implementación de estudio base para especies forestales amenazadas en el departamento del Chocó», el cual contempló el levantamiento de información secundaria y datos cualitativos y cuantitativos de cinco especies forestales amenazadas, jigua negro (O. cernea), guayaquil (C.paraense), abarco (C.pyriformis), pino amarillo (Podocarpus sp.) y guayacán amarillo (Tabebuia chrysantha) en cuatro municipios del departamento del Chocó, Juradó, Istmina, Río Quito y Riosucio, particularmente en territorios de los Cabildos Indígenas
Mayor de Juradó y Nassipurrú en Juradó, el Consejo Comunitario Mayor de Istmina, la Comunidad de San Isidro en Río Quito y el Consejo Comunitario de Truandó y Medio en Riosucio.

Área de estudio.El estudio se desarrolló en el municipio de Riosucio, en los consejos de comunidades afrocolombiana del río Salaquí. El territorio del Consejo Comunitario Mayor de la Cuenca del río Salaquí se encuentra localizado en la parte baja del río Atrato, al noroccidente colombiano y en la parte norte del departamento del Chocó, entre las coordenadas $7^{\circ} 16^{\prime} 00^{\prime \prime}-7^{\circ} 30^{\prime} 00$ latitud $\mathrm{N}$ y $77^{\circ} 06^{\prime} 00^{\prime \prime}-77^{\circ} 36^{\prime} 00^{\prime \prime}$ de longitud oeste (Figura 1).

\section{Metodología}

El estudio se realizó teniendo en cuenta los criterios establecidos en el Decreto 1791 de 1996, la Guía Técnica del MMA, la Resolución 987 de 2000 o Estatuto Forestal de CODECHOCO (2000), además de ciertos elementos tomados de experiencias regionales. Se planteó el seguimiento a las 8 resoluciones de aprovechamiento otorgadas por CODECHOCO a los consejos menores de la cuenca (Playa Aguirre, Caño Seco, Salaquisito, San José de Tamboral, Villa Nueva Tamboral, Arenal Medio, Playa Bonita y la otorgada al Consejo Mayor). Se realizaron recorridos por el área para identificar los sitios donde se establecieron las parcelas de monitoreo; se utilizó un GPS que permitió su georeferenciación, se delimitaron 6 parcelas de $50 \mathrm{~m}$ x $50 \mathrm{~m}$ $=2500 \mathrm{~m}^{2}$ equivalentes a 0.25 ha las cuales quedaron distribuidas en 4 consejos comunitarios locales de la cuenca del río Salaquí; cada parcela fue dividida en 25 subparcelas de $10 \mathrm{~m} \times 10 \mathrm{~m}$ donde se midió el diámetro a todos los aboles de las especies seleccionadas a la altura del pecho (DAP) $\geq 10 \mathrm{~cm}$, se estimaron las alturas de cada árbol y de los pisos del bosque cuando fue posible, además se realizó el análisis de la estructura horizontal lo que permite realizar una evaluación de los árboles individualmente y de las especies en la superficie del bosque lo cual fue evaluado a través de los índices, los cuales expresan la ocurrencia de estas especies al igual que la importancia ecológica al interior del ecosistema, es el caso de las abundancias, frecuencias y dominancias cuya sumatoria relativa genera el índice de valor de importancia. De igual forma se utilizó el programa EXCEL para el procesamiento de la información. Los índices de diversidad Shannon-Weaver (H), Margalef y el de equidad asociado, se calcularon mediante el Past Program (Figura 2).

\section{Resultados y discusión}

De acuerdo con los datos obtenidos con los diferentes madereros de la zona, las maderas provenientes de la cuenca del Salaquí son amparadas por resoluciones de otros conse- 


\section{Bioetnia Volumen 9 № 2 (julio-diciembre), 2012}

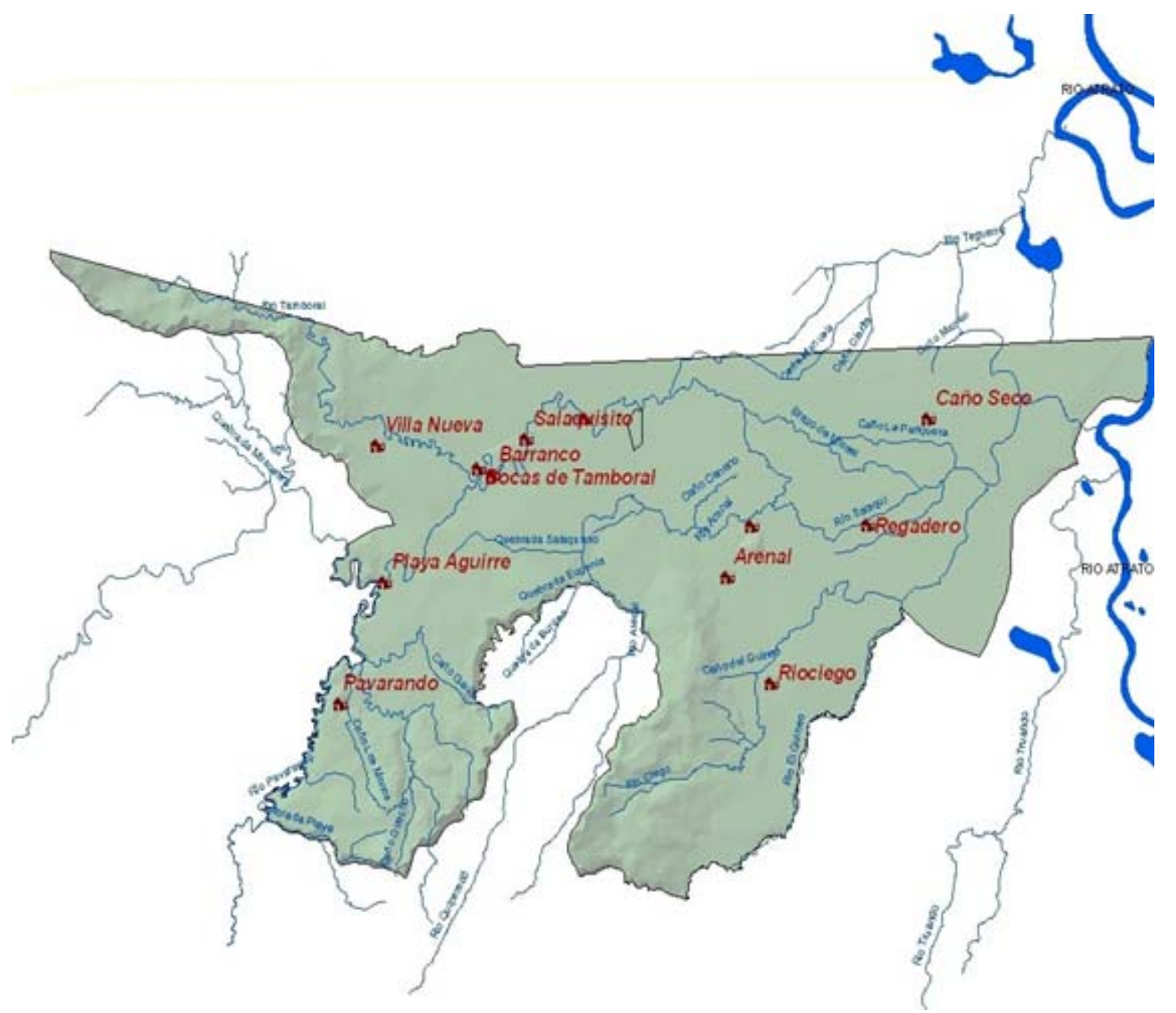

Figura 1. Ubicación del área de estudio cuenca del río Salaquí

jos comunitarios ubicados alrededor del municipio los cuales tienen autorizaciones vigentes; cabe anotar que de los 85.694 $\mathrm{m}^{3}$ otorgados para la cuenca del río Salaquí entre los años 2007 y 2012, de los cuales $64.149 \mathrm{~m}^{3}$ corresponden a especies que se encuentran en estado vulnerable y otras en peligro de extinción, como es el caso del abarco, roble, caracolí, santa cruz, polvillo, choibá, bálsamo, cativo, olleto, guino, especies que están dentro del grupo de estudio. De acuerdo con los datos de movilización el abarco es la especie más movilizada contribuyendo así al agotamiento de la misma (Figura 3).

Mediante la realización del presente estudio se establecieron tres puntos para el control de las especies movilizadas teniendo como punto principal el que se encuentra ubicado en la desembocadura del río Salaquí por ser esta nuestra zona de influencia (Figura 4).

Composición florística. En el muestreo realizado en un sector de bosque de la cuenca del río Salaquí, se registraron
372 individuos los cuales se distribuyeron en 7 familias, 14 especies y 13 géneros. De acuerdo con estos resultados, en la parcela I se registraron 57 individuos, representados en 6 familias, 9 especies, siendo las familias más representativas Fabaceae con 3 especies y Anacardiaceae con 2 especies; en esta parcela la familia Fabaceae estuvo representada por las especies Myroxylum balsamun con 2 individuos, Dypterix oleifera con 9 individuos y Centrolobium paraence con 3 individuos. Anacardiaceae con las especies Anacardium excelsum con 12 individuos, Astroniun graveolens con 12 individuos. Bignoniaceae estuvo representada por la especie Tabebuia serratifolia con 7 individuos, Caesalpinaceae representada por la especie Peltogyme porphycordia con 6 individuos, Lauraceae con la especie Nectandra membranaceae con 2 individuos y Lecitidaceae, con 4 individuos que pertenecen a la especie Lecytis sp. (Tabla 1).

Análisis de estructura horizontal. De acuerdo con la información obtenida en el censo forestal se encontró que la 


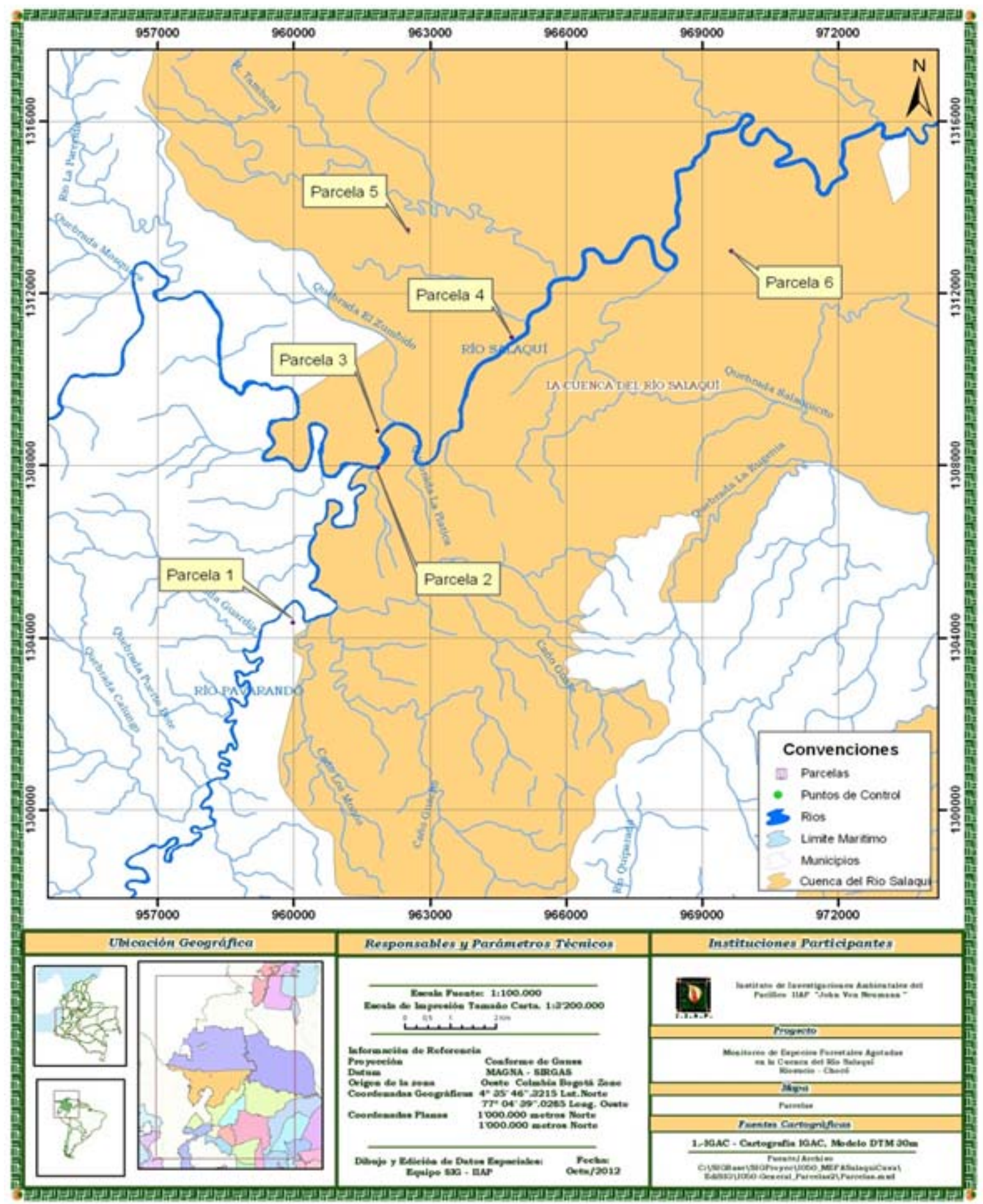

Figura 2. Ubicación geográfica de las parcelas Monitoreo en la cuenca del río Salaquí

especie más abundante es el guayaquil seguida de caracolí y olleto, mientras que la especie más escasa fue el bálsamo. Se encontró gran cantidad de individuos en las clases diamétricas intermedias lo que nos indica que no se encuentran bien distribuidas en el área censada, sino que se agrupan en manchas o conglomerados, los cuales se encuentran frecuentes en la naturaleza esto a causa de las variaciones ambientales relativamente pequeñas pero importantes para los individuos que integran dicha población. Los bosques tropicales naturales son muy heterogéneos y están conformados por 
Bioetnia Volumen 9 № 2 (julio-diciembre), 2012

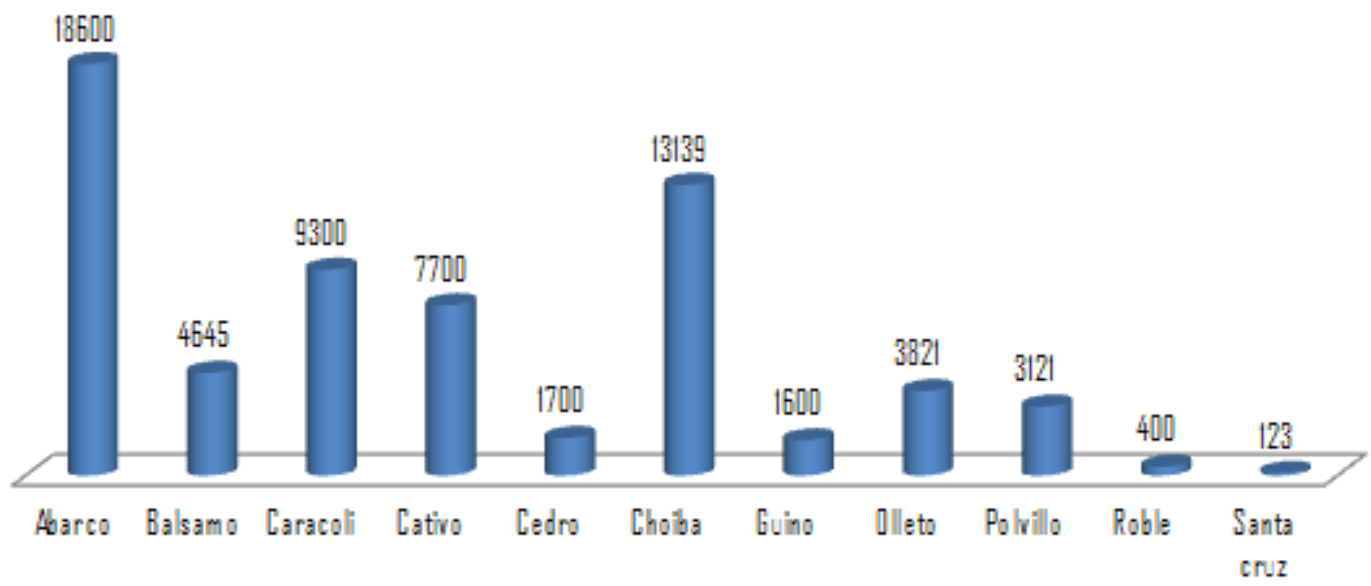

Figura 3. Datos de las especies más movilizadas en la cuenca del río Salaquí desde el año 2007 hasta el 2012

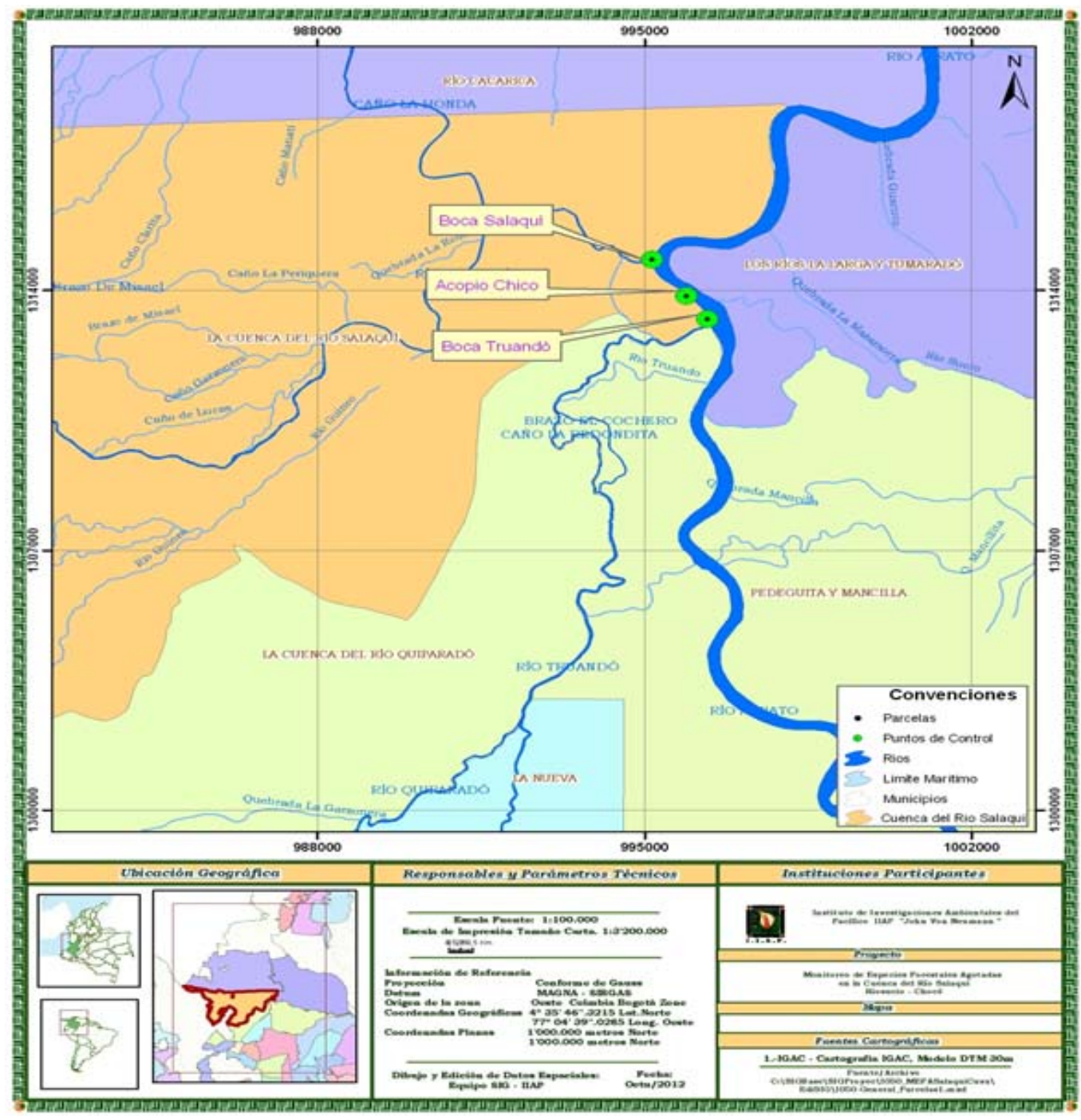

Figura 4. Puntos de monitoreo 
Tabla 1

Composición florística

\begin{tabular}{|c|c|c|c|c|c|c|}
\hline $\mathbf{N}^{\circ}$ & Especies & Nombre científico & Familia & $\begin{array}{c}\mathbf{N}^{\circ} \text { de } \\
\text { árboles }\end{array}$ & $\begin{array}{l}\text { Área } \\
\text { basal }\end{array}$ & Volumen \\
\hline 1 & Abarco & Caryniana pyriformis & Lecitidaceae & 9 & 0,80 & 0,27 \\
\hline 2 & Bálsamo & Myroxylum balsamun & Fabaceae & 2 & 0,38 & 0,53 \\
\hline 3 & Caidita & Nectandra menmbranacea & Lauraceae & 17 & 2,07 & 0,87 \\
\hline 4 & Caracolí & Anacardium excelsum & Anacardiaceae & 40 & 55,50 & 63,21 \\
\hline 5 & Cativo & Prioira copaifera & Caesalpinaceae & 28 & 9,35 & 7,09 \\
\hline 6 & Cedro & Cedrela odorata & Meliaceae & 17 & 2,52 & 0,88 \\
\hline 7 & Choiba & Dypterix panamensis & Fabaceae & 71 & 143,63 & 204,76 \\
\hline 8 & Guayaquil & Centrolobium paraence & Fabaceae & 72 & 8,51 & 3,12 \\
\hline 9 & Guino & Carapa guianensis & Meliaceae & 3 & 1,35 & 0,74 \\
\hline 10 & Nazareno & Peltogyme porphyrocardia & Caesalpinaceae & 6 & 4,06 & 2,96 \\
\hline 11 & Olleto & Lecytis sp. & Lecitidaceae & 48 & 70,11 & 84,13 \\
\hline 12 & Polvillo & Tabebuia erratifolia & Bignoniaceae & 24 & 8,42 & 4,56 \\
\hline 13 & Roble & Tabebuia rosea & Fabaceae & 23 & 1,60 & 0,39 \\
\hline 14 & Santa cruz & Astroniun graveolens & Anacardiaceae & 12 & 8,14 & 5,55 \\
\hline & Total general & & & 372 & 316,42 & 379,04 \\
\hline
\end{tabular}

gran diversidad de especies, con diferentes edades dentro de las cuales, según la FAO, se diferencian los tres estados sucecionales a saber brinzal, latizal y fustal.

Abundancia de especies. Del total de los individuos distribuidos en las parcelas muestreadas, el guayaquil ( $C$. paraence) fue la especie más abundante con $72(19,4 \%)$ individuos, seguida del choibá y olleto (Lecytis sp.) con 48 $(12,9 \%)$ individuos, el caracolí (A. excelsum) con $40(10,8 \%)$ individuos entre otras. La especie menos abundante en el estudio correspondió a la especie bálsamo (Myroxilum balsamum) con $2(1,9 \%)$ individuos.

Frecuencia de especies. Las especies más frecuentes en el estudio efectuado en la cuenca del río Salaquí son Anacardium excelsum, Dypterix oleifera, Centrolobium paraence; mientras que la menos frecuente fue la especie Myroxilum balsamum, de acuerdo con los histogramas de frecuencia los cuales permiten la evaluación de la estructura horizontal en los ecosistemas boscosos, que se generan a partir de la agrupación de las especies en categorías o clases de frecuencia absoluta.

De acuerdo con los resultados obtenidos en el histograma de las frecuencias de las especies estudiadas podemos concluir que el bosque tiende a la heterogeneidad, debido a que se encuentran valores altos en las clases dimétricos superiores.

Dominancia de especies. El estudio concluye que la familia Fabaceae $(45,2 \%)$ como la más dominante, seguida de la familia Lecitidaceae $(19,4 \%)$ y la familia Caesalpinaceae $(17,5 \%)$. La familia que presentó la menor dominancia fue Lauraceae $(0,27 \%)$. A través del análisis obtenido podemos definir que la parcela que presentó mayor dominancia fue la parcela 5 cuya familia más dominante fue Fabaceae lo cual corrobora la dominancia de esta familia en el desarrollo del presente estudio.

Índice valor de importancia (IVI). Las especies que reportaron el IVI más alto en las 6 parcelas monitoreadas fueron, A. excelsum, C. paraence, Dypterix sp., Lecytis sp., estas forman el conjunto de especies principales, lo que indica que existen condiciones para su recuperación porque a través de tratamientos silviculturales se puede realizar repoblamiento y dispersión de las especies más amenazadas (Centrolobium paraence y Cariniana pyriformis). El valor del área basal determinó que A. excelsum, Lecytis sp., Dypterix sp. poseen los IVI más elevados y además de ello hacen presencia en todas las parcelas muestreadas (Tabla 2).

Estructura vertical. En los bosques de la cuenca del río Salaquí se presentan alturas hasta de más de $30 \mathrm{~m}$, en el cual se distinguen varios estratos arbóreos, los cuales se pueden clasificar como alto, medio y bajo. El estrato alto estuvo constituido por alturas mayores a $30 \mathrm{~m}$, en este se registraron 4 individuos los cuales no hacen parte de las especies objeto 
Bioetnia Volumen 9 № 2 (julio-diciembre), 2012

Tabla 2

Volumen por parcela

\begin{tabular}{lrrrrrrr}
\hline Clases DAP & Rango $(\mathbf{c m})$ & \multicolumn{7}{c}{ Parcela } \\
& & $\mathbf{1}$ & $\mathbf{2}$ & $\mathbf{3}$ & $\mathbf{4}$ & $\mathbf{5}$ & $\mathbf{6}$ \\
I & & & & & & \\
II & $10,0-25.9$ & 0,001 & 0,043 & 0,026 & 0,062 & 0,043 & 0,018 \\
III & $25,9-39,9$ & 0,036 & 0,216 & 0,114 & 0,163 & 0,194 & 0,115 \\
IV & $39,9-59,0$ & 0,405 & 0,444 & 0,347 & 0,701 & 0,478 & 0,177 \\
V & $59,0-69,4$ & 0,468 & 0,401 & 0,421 & 0,453 & 0,777 & 0,356 \\
Total & $>70$ & 35,082 & 37,719 & 37,927 & 138,308 & 101,487 & 22,770 \\
\hline
\end{tabular}

de estudio pero se encontraron en su zona de influencia; el individuo con mayor altura $(32 \mathrm{~m})$ pertenece a la especie ceiba, pero algunas de las especies estudiadas alcanzan alturas similares como es el caso del Lecythis sp., Myroxylum balsamun y el Tabebuia serratifolia con $30 \mathrm{~m}$. En el estrato medio se ubican individuos con alturas entre los 15 y $27 \mathrm{~m}$ en la cual se relacionan especies como A. excelsum, Dypterix sp., Tabebuia serratifolia, T. rocea, Astroniun graveolens, Centrolobium paraence seguidas por Nectandra menbranaceae, Peltogyne porphycordia. En el estrato más bajo formado por individuos entre los 3 y los $14 \mathrm{~m}$ se encontró un gran número de individuos de las especies $C$. paraence, $T$. serratifolia, lo que hace favorable el establecimiento de una propuesta para su manejo y conservación, porque son especies que se encuentran en estado crítico.

La estructura vertical del bosque presenta el mismo patrón con el que se expresa la estructura diamétrica; se pudo observar que gran parte de los individuos se encuentran en estado de crecimiento, dato que se asemeja a los reportados para San José del Palmar por Devia et al. (1999) donde se afirma que la mayor parte de los individuos son de tamaño mediano a pequeño, ubicándose en los estratos I y II. Esto sumado a lo expresado en estudios realizados en bosques naturales del Valle por García et al. (2010) donde expresan que la mayoría de los árboles están esperando mejores condiciones de luminosidad para alcanzar su desarrollo y ascender al dosel, además con los estudios realizados por Galeano (2001) en estribaciones del golfo de Tribugá en el Chocó, donde reportan que $86 \%$ de los individuos $\geq 10 \mathrm{~cm}$ DAP no superan los $25 \mathrm{~m}$ de altura, ubicándolos en las primeras categorías altimétricas. La altura promedio registrada para la zona fue de $25 \mathrm{~m}$, este valor muestra gran similitud con los reportados por Cleef et al. (1984) para la cuenca del río Buriticá, la cual oscila entre 25 y 30 m; a pesar de esto se alcanza a denotar que gran parte de las especies no presentan individuos en los rangos de 20 a $39 \mathrm{~cm}$ de DAP esto puede ser causado por el alto grado de intervención al que están siendo sometidos los bosques naturales y por esta causa se denotan manchas en algunas áreas con elementos en regeneración. La concentración de individuos y especies en el sotobosque y la disminución drástica de estos a medida que se van desarrollando para alcanzar el dosel; parece ser este un patrón común en los bosques tropicales (Gómez-Pompa et al., 1971 citado por Galeano, 2001) este fenómeno pudo ser observado en las parcelas 2 y 5 donde se encontró un gran número de individuos y especies localizadas en las primeras categorías diamétricas y de altura formando una «J» invertida.

Indicadores de diversidad. A partir de la información obtenida se elaboraron curvas de especies-área para cada parcela, las cuales consistieron en graficar el número de especies vegetales encontradas para una superficie de muestreo determinada (Greig-Smith, 1983). El índice de Margalef indica que los valores más altos de riqueza se registraron en las parcelas uno y dos con 1,98 y 1,96; al igual denota que los valores más bajos fueron arrojados por las parcelas cinco y tres $(1,69$ y 1,70$)$; a nivel del área de estudio, Margalef reportó un valor de 10,95, indicando así que los bosques de la cuenca del río Salaquí poseen una alta riqueza.

Shannon-Weaver basado en abundancia relativa de las especies evaluadas para este estudio muestra que los valores máximos fueron reportados en las parcelas dos $(2,09)$ y uno $(2,02)$ lo que nos indica que hay una alta diversidad de especies teniendo en cuenta el área evaluada (1,5 ha); el valor reportado por Shannon-Weaver para todas las parcelas fue 11,63. Los índices utilizados Shannon-Weaver, equidad (E), calculados respectivamente en el programa Past versión 1,15 . Hammer y Harper (2003) informan valores muy similares en las diferentes parcelas, lo que determina el alto grado de heterogeneidad que posee la cuenca del río Salaquí y demuestra que es una de las zonas más ricas y biodiversas del Chocó Biogeográfico. Es de anotar que las especies que se encuentran presentes en las unidades de muestreo son muy apropiadas para el monitoreo y seguimiento a través de la 


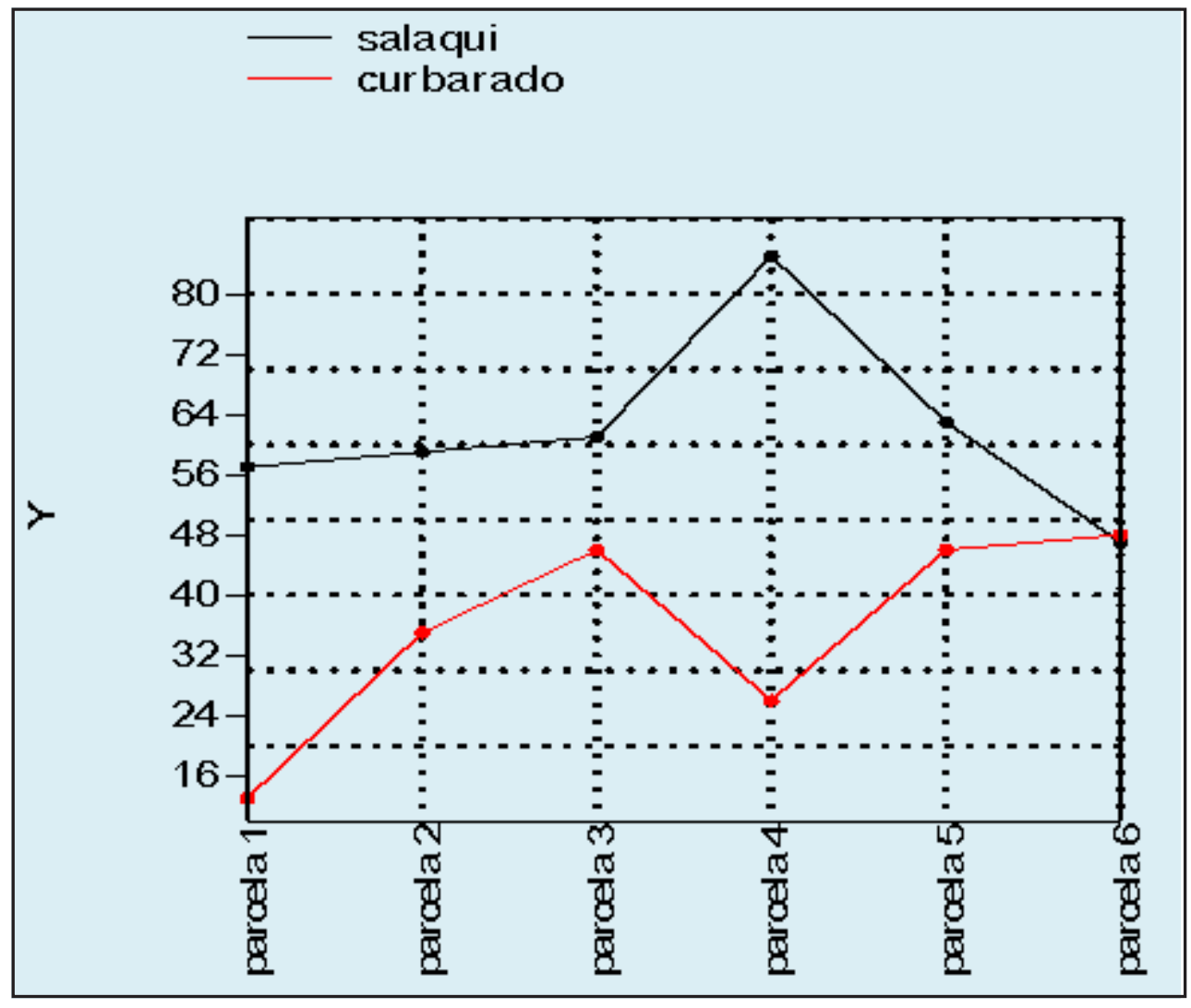

Figura 5. Comparativo de diversidad entre Salaquí y Curvaradó

implementación de parcelas permanentes de investigación las cuales permitirían la conservación de las mismas especies. En relación con el número de árboles por parcela, las que presentaron mayor número de individuos fueron las parcelas 4 (88), 5 (63) y 3 (61).

La riqueza para las especies muestreadas en el bosque de la parte alta y media de la cuenca del río Salaquí, considerando solo aquellos individuos con DAP $\geq 10 \mathrm{~cm}$ fue relativamente alta en relación con los estudios realizados en otros bosques del departamento del Chocó, como en la cuenca del río Curvaradó el cual posee la misma cantidad de área estudiada (1,5 h) (Figura 5) en la que se alcanzó un promedio de 35 individuos los cuales se distribuyen en 5 especies y 4 familias con DAP $\geq 10 \mathrm{~cm}$ (IIAP, 2012), mientras que el promedio alcanzado para la cuenca del Salaquí que fue de 15 especies y 7 familias; cabe destacar que la cuenca del río Curvaradó está entre la llanura aluvial de los ríos Curvaradó y Atrato, se registraron menos especies y familias para la cuenca del río Salaquí, además muestra mayor homogeneidad que otras zonas del Chocó. Las familias fueron comunes para las seis unidades de muestreo, lo que muestra una buena distribución de las especies en el sitio; cabe destacar que la familia Fabacea representa las especies con mayor importancia ecológica para la zona.

Autores como García et al. (2004), en estudios realizados en otros sectores del Chocó reportan que las familias más abundantes y frecuentes son las Erecaceas seguida por la Sapotaceas, Lecithydacea, Clusiaceae, Euphorbiacea, Moraceae, Caesalpinaceae, Fabaceae, Mimosacea, coincidiendo así con lo afirmado por Devia et al. (1994) lo cual difiere notablemente con los resultados obtenidos en el presente estudio en condiciones de topografía, altura y al alto grado de extracción de las especies forestales maderables. En conclusión las familias Fabaceae, Anacardiaceae, Lecythidaceae, Lauracea son las más importantes en el sector de bosque evaluado debido a que representan a las especies Centrolobium paraence, Anacardium excelsum, Lecytis sp., Nectandra membranacea, que ejercen el dominio en cada uno de los sitios muestreados lo que nos demuestra que a pesar de estar en estado de agotamiento se pueden mejorar las condiciones para su sostenimiento y conservación por intermedio de prácticas de manejo sostenible.

Es de anotar que a pesar del alto grado de intervención antrópica, los bosques de la cuenca del río Salaquí aún posee 


\section{Bioetnia Volumen 9 № 2 (julio-diciembre), 2012}

una gran riqueza de especies, situación que resulta relevante para continuar con la realización de estudios de diversidad florística y con la implementación de planes de manejo encaminados a su sostenimiento, conservación y posterior aprovechamiento garantizando la preservación de las especies, lo cual genera alternativas económicas y ambientales, es por ello que se sugiere el seguimiento a largo plazo mediante el monitoreo de las parcelas permanentes establecidas en dicha cuenca hidrográfica.

Diversidad de especies. El índice de Shannon-Weaver mostró que entre las parcelas estudiadas existen diferencias significativas entre las parcelas 2-5 y 2-6 presentando los mayores valores las parcelas $2(2,09)$ y $1(2,02)$ y un menor valor en la parcela $6(1,79)$, obteniendo un promedio general para el área de estudio de 1,94 mostrando valores muy similares para las parcelas y demuestra la alta heterogeneidad que presenta la cuenca del río Salaquí lo que difiere con lo expresado por Shannon-Weaver en el estudio similar realizado en la cuenca del río Curvaradó, el cual mostró diferencias altamente significativas entre las parcelas 6-1 y 6-4, donde el máximo valor para Shannon lo obtuvieron las parcelas $6(1,56)$ y $5(1,49)$, el valor de Shannon-Weaver para las parcelas de la cuenca de Curvaradó fue 1,38, lo que demuestra la gran diversidad ecológica que posee la cuenca del río Salaquí. Margalef indica que los bosques de la cuenca del río Salaquí $(10,95)$ poseen mayor riqueza que los de la cuenca de Curbaradó $(6,06)$. Es de anotar que esta cuenca ha recibido una mayor intervención antrópica y sus coberturas boscosas están a punto de desaparecer.

Cálculos entre volumen por parcela. Se calcularon los volúmenes de los individuos inventariados con el objeto de conocer el potencial volumétrico de cada una de las especies teniendo en cuenta las categorías diamétricas, las cuales arrojaron un volumen $\left(\mathrm{m}^{3}\right)$ de madera que para estas parcelas fue de $379,04 \mathrm{~m}^{3}$ en el cual no se tuvo en cuenta otro tipo de vegetación como palmas o arvenses donde el mayor volumen se obtuvo en la parcela $4\left(139,69 \mathrm{~m}^{3}\right)$ seguido de la parcela $5\left(102,96 \mathrm{~m}^{3}\right)$, la parcela $3\left(38,84 \mathrm{~m}^{3}\right)$ la parcela $2\left(38,82 \mathrm{~m}^{3}\right)$, la parcela $1\left(35,51 \mathrm{~m}^{3}\right)$ y la parcela $6(23,20)$.

\section{Conclusiones}

Los avances alcanzados por los representantes de los Consejos Comunitarios Locales se han visto determinado por el apoyo que reciben de entidades como el IIAP y CODECHOCÓ porque han logrado un alto grado de unificación al interior de los mismos. Esto ha traído como consecuencia que se disminuya el aprovechamiento de forma ilegal como se venía realizando porque para realizar esta actividad en los diferentes consejos menores deben contar con el aval otorgado por el respectivo represente legal del consejo en el cual se desarrollará la actividad. Se cuenta con un equipo técnico consolidado lo que contribuye a una buena gestión y está constituido por 10 personas y es liderado por el representante legal de la cuenca y cuenta con el apoyo de CODECHOCÓ y la policía; los asesores necesitan continuar con el proceso, pues, a través de la consolidación de este comité se contribuye con la disminución de la ilegalidad y se generan alternativas reales que ayuden a la conservación de los bosques y demás ecosistemas.

\section{Agradecimientos}

El autor expresa sus agradecimientos al Instituto de Investigaciones del Pacífico (IIAP) y a su director William Klinger, por la oportunidad de ingresar a esta institución; a Luis Alirio Córdoba, Representante legal de la cuenca del río Salaquí y su Junta Directiva, a Giovanny Ramírez Moreno y Zulmary Valoyes por creer en mis capacidades y además de brindarme su apoyo, confianza y paciencia durante la ejecución del proyecto.

\section{Literatura citada}

Cleef, A., O. Rangel-Ch, T. Van der Hammen, R. Jaramillo. 1984. La vegetación de las selvas del transecto Buritacá. En: Van der Hammen y Ruíz, (Eds). La Sierra Nevada de Santa Marta(Colombia). Transecto BuritacáLa Cumbre. Estudios de Ecosistemas Tropandinos. 2. Berlín: J. Cramer.

CODECHOCÓ. 2000. Resolución 987 del 2000. Estatuto forestal. En línea. [fecha de acceso 15 de agosto de 2012]. Disponible en: http// www.senado.gov.co/sala-de.../51-050legislacionenmateriade-bosques

Devia, A., D. Cárdenas, A. Cogollo. 1994. Contribución al estudio florístico de la reserva natural del río Escalerete, Buenaventura, Colombia. En: Memorias del I Congreso Nacional sobre Biodiversidad. Proyecto Biopacífico. Cali: Universidad del Valle, Instituto de Estudios del Pacífico. 77-84 pp.

Devia, A., C. M. Taylor, A. Cogollo, J. Adarve. 1999. Vegetación florística de la reserva del río Escalerete, región Biogeográfica del Chocó, Valle, Colombia. Datos preliminares En: O. Rangel-Ch et al. (Eds.). Libro de Resúmenes I Congreso Colombiano de Botánica. Bogotá: Instituto de Ciencias Naturales. Universidad Nacional de Colombia. 22 pp.

Galeano, G. 2001. Forest use at the Pacific coast of Chocó, Colombia: A quantitative approach. Economic Botany. 54 (3): pág iniciales y finales?

García, C., M. Moreno, D. Robledo, L. Mosquera, D. Palacios. 2004. Composición y diversidad florística de los bosques de la cuenca hidrográfica del río Cabí, Quibdó, Chocó. Rev Institucional Investigación, Biodiversidad y Desarrollo. 20: 13-23

García, M., R. Haack, R. Magarey, M. Margosian. 2010. Modeling spatial establishment patterns of exotic forest insects in urban areas in relation to tree cover and propagule pressure. J Econ Entomol. 103 (1): 108-18.

Gentry, A. 1989. Changes in plant community diversity and floristic composition on environmental and geographical gradients. An Missouri Bot Garden. 75: $1-74$.

Gómez-Pompa, A. 1971. Posible papel de la vegetación secundaria en la evolución de la flora tropical. Biotropica. 3: 125-35.

Greig-Smith, P. 1983. Quantitative plant ecology. Berkeley: University of California Press. 359 pp.

IIAP. 2009. Especies forestales amenazadas en 5 municipios del Chocó Biogeográfico (Riosucio, Istmina, Río Quito, Jurado y Carmen del Darién). Quibdó: Instituto de Investigaciones Ambientales del Pacífico.

IIAP. 2009. Implementación de estudio base para especies forestales amenazadas, en el departamento del Chocó, municipio deAalto Baudó. Quibdó: Instituto de Investigaciones Ambientales del Pacífico. 
Monitoreo de especies forestales nativas en la cuenca del río Salaquí. RS. Mosquera

IIAP. 2012. Especies forestales amenazadas en la cuenca del río Curvaradó, municipio Carmen del Darién. Quibdó: Instituto de Investigaciones Ambientales del Pacífico.

Ministerio del Medio Ambiente. Decreto 1791 de 1996, República de Colom- bia. Bogotá. En línea [fecha de acceso: 20 agosto de 2012] disponible en URL: http//: http://www.alcaldiabogota.gov.co/sisjur/normas/ Norma1.jsp?i=1296 